\title{
Was können NSAR, was TNF-a-Inhibitoren nicht können?
}

\author{
Nichtsteroidale Antirheumatika sind das First-Line-Medikament für Patienten mit axialer Spondylarthritis. Welche \\ Effekte sie auf die Pathologie entfalten und wie diese sich von denen der TNF-a-Inhibitoren unterscheiden, war \\ Thema beim Deutschen Rheumatologenkongress 2014.
}

\begin{abstract}
Der Großteil der Patienten mit axialer Spondylarthritis (axSpA) kann effektiv mit Nichtsteroidalen Antirheumatika (NSAR) behandelt werden. Sie sind die Eckpfeiler der medikamentösen Therapie. Aber auch diagnostisch sind sie hilfreich. Denn wenn Patienten mit chronischem Rückenschmerz innerhalb von 24-48 $\mathrm{h}$ auf die Therapie ansprechen, unterstützt das die Diagnose axSpA, wie Prof. Dr. Jürgen Braun, Medizinischer Direktor des Rheumazentrums Ruhrgebiet, Herne, anlässlich der Sitzung „What is new: SpA" berichtete. „Eine mechanische Ursache für den Rückenschmerz ist dann so gut wie ausgeschlossen." NSAR wirken also gut auf den typischen entzündlichen Rückenschmerz. Das gilt vor allem, wenn sie kontinuierlich und nicht nur bei Bedarf eingenommen werden. Die kontinuierliche Einnahme hat noch einen weiteren Vorteil, wie Braun in Erinnerung rief: Die Röntgenprogression wird vor allem bei Patienten mit deutlich erhöhten Entzündungsmarkern gehemmt.

TNF-a-Inhibitoren reduzieren bei Patienten mit axSpA ebenfalls effektiv die klinische Schmerzsymptomatik. Darüber hinaus nehmen sie Einfluss auf die Wirbelsäulenbeweglichkeit und damit die körperliche Funk-
\end{abstract}

tionsfähigkeit. Anders als NSAR tragen TNF-a-Inhibitoren auch zur Senkung der CRP-Spiegel bei. Und sie reduzieren erwiesenermaßen hochsignifikant die Entzündungsaktivität im Achsenskelett, wie mittels Magnetresonanztomographie gezeigt werden kann. Auf die Röntgenprogression scheinen sie, zumindest in den ersten Jahren, keinen wesentlichen Effekt zu haben. Braun gab allerdings zu bedenken, dass ein Direktvergleich der Effekte von NSAR und TNF-a-Inhibitoren nicht möglich sei, weil letztere erst als Second-Line-Medikamente eingesetzt werden.

\section{Welches NSAR für wen?}

Bei der Wahl des NSAR riet Braun, auf die Verträglichkeit und bei vorwiegend nächtlichen Schmerzen auf die Halbwertzeit zu achten. Bei der gewählten Dosis sollte bedacht werden, dass diese nicht nur für den Schmerz von Bedeutung ist. Bekanntermaßen führt die höhere Dosis von Etoricoxib eher zur Remission, die von Celecoxib lindert die Morgensteifigkeit stärker.

Aufgrund befürchteter oder tatsächlicher Nebenwirkungen nehmen viele axSpA-Patienten nicht die emp- fohlene maximale NSAR-Dosis. Daher kann es laut Braun sinnvoll sein, mit der Intensivierung der Therapie auf TNF-a-Inhibitoren etwas länger als vier Wochen zu warten. Denn gerade Patienten mit Syndesmophyten und hohen Serumwerten des C-reaktiven Proteins zu Beginn profitieren, zumal wenn sie männlich und Raucher sind, im Sinne der Inhibition der Knochenneubildung von einer NSAR-Therapie.

Zukünftig könnte die Kombination beider Wirkmechanismen eine Alternative sein. Wie Sieper et al. in ihrer kürzlich publizierten INFAST-Studie (Sieper J et al (2014) Ann Rheum Dis 73(1):101-107) zeigen konnten, erzielten nach 28 Wochen doppelt so viele axSpA-Patienten unter der Kombinationstherapie aus Naproxen plus Infliximab eine partielle Remission.

Dr. Wiebke Kathmann

Quelle:

springermedizin.de basierend auf: „What is new: SpA", DGRh Kongress 2014

rheuma plus $2014 \cdot 13: 10$

DOI 10.1007/s12688-014-0006-y

Online publiziert: 14. November 2014

(อ) Springer-Verlag Wien 2014 